Rev. Saúde públ. S. Paulo

7: $161-79,1973$

\title{
ANÁlISE DE DADOS SOCIO-ECONOMMICOS E SANITÁRIOS DE FAMÍLIAS RESIDENTES NO DISTRITO-SEDE DE BOTUCATU (SÃO PAULO, BRASIL), EM 1969 *
}

Maria Iraci Cabianca RAMOS **

Mário A. Carneiro Leäo RIBEIRO ***

Cecília MAGALDI **

Massako IYDA **

Eurivaldo Sampaio de ALMEIDA **

Nelson de SOUZA **

RSPU-B $/ 169$

RAmos, M. I. C. et al. Análise de daăos sócio-econômicos e sanitários de familias residentes no distrito-sede de Botucatu (São Paulo, Brasil), em 1969. Rev. Saúde públ., S. Paulo, 7: 161-79, 1973.

Resumo: Foi realizado, em 1969, um levantamento de dados sócio-econômicos e sanitários da zona urbana de Botucatu. Trezentas e cinco familias amostradas foram entrevistadas sobre: constituição familiar, idade, ocupação, renda, grau de instrução, condições sanitárias dos domicilios, conhecimento $e$ uso de vacinas, demanda $e$ opiniōes relativas aos serviços de saude. Algumas respostas foram associadas ao "status" sócio-económico das familias. As condiçôes de saneamento básico estavam dentro de padrões quantitativos razoáveis $e$ era alto o desconhecimento sobre o valor $\boldsymbol{e}$ numero de doses das vacinas. Constatou-se acentuada dispersão de recursos para saude, indicando falta de coordenação dos orgãos assistenciais; a demanda ao Centro de Saude era baixa, quase restrita às crianças, indicando distorsão da percepção da comunidade com relação aos serviços médico-sanitários e necessidade de desenvolvimento adequado desses serviços, além de educação sanitária sistemática.

UNITERmos: Inquérito sócio-econômico (Botucatu, SP, Brasil) *; Saneamento do meio *; Recurso saúde*; Ciências sociais aplicadas.

$$
1 \text { - I N T R O D U Ç A O }
$$

Um dos requisitos fundamentais para que a universidade possa desempenhar seu papel de instrumento básico de transformação social pelo qual, necessariamente, devem ser adequadas suas atividades e influências à realidade onde se insere, consiste no conhecimento, o tanto quanto possível amplo e profundo, do sistema social e seus sub-sistemas. Esta necessidade impõe-se ainda mais

* Apresentado na 2.a Jornada Científica da Faculdade de Ciências Médicas e Biológicas de Botucatu, em 1972.

* Do Departamento de Medicina Preventíva, Social e Saúde Pública da Faculdade de Ciências Médicas e Biologicas de Batucatu - Botucatu, SP-Brasil.

** Do Departamento de Matemática da Faculdade de Ciências Médicas e Biológicas de Botucatu - Botucatu, SP-Brasil. 
RAMOS, M. I. C. et al. - Análise de dados sócio-econômicos e sanitários de famíllas residentes no distrito-sede de Botucatu (São Paulo, Brasil), em 1969. Rev. Saúde públ., S. Paulo, 7: 161-79, 1973.

na área da saúde, partindo-se da premissa aceita por muitos de que a escola médica através de serviços prestados no hospital universitário e diretamente em nível comunitário esteja integrada no sistema de saúde local e/ou regional.

Neste sentido, era desejável que, ao lado de outros levantamentos setoriais, a cargo da Faculdade de Ciências Médicas e Biológicas de Botucatu (FCMBB) e de outras entidades, dispuséssemos de alguns dados sócio-econômicos, sanitários e relativos à prestação de serviços de saúde, obtidos de forma preliminar, através de entrevista de uma amostra representativa da população urbana.

Além disso, pretendia-se, com os dados levantados obter elementos informativos quanto ao conhecimento, opinião e aceitação de serviços de saúde, que pudessem servir de orientação e subsídio para instalação do Centro de Saúde Escola da FCMBB em uma área urbana de Botucatu (em convênio com a Secretaria da Saúde do Estado de São Paulo), e consequientemente para o planejamento a ser elaborado.

Nosso terceiro objetivo era oferecer, através de pesquisa desde sua fase de planejamento propriamente dita, uma oportunidade para treinamento em trabalho de campo, na área de saúde, aos alunos que cursavam a disciplina de Ciências Sociais Aplicadas à Medicina, em 1969 (Departamento de Medicina Preventiva, Social e Saúde Pública da FCMBB).

\section{1 - Informações sobre o Municipio de Botucatu}

O município de Botucatu localiza-se quase no centro geográfico do Estado de São Paulo, pertencendo à região administrativa de Sorocaba (sub-região 4-7). Compreende uma área de $1522 \mathrm{Km}^{2}$, com altitude média de $885 \mathrm{~m}$. Situa-se a $22^{\circ} 52^{\prime} 20^{\prime \prime}$ de latitude sul e $48^{\circ} 26^{\prime} 38^{\prime \prime}$ de longitude WGR.

Compõe-se de três distritos: Botucatu (distrito-sede onde foi realizada a pesquisa), Rubião Junior e Vitoriana. O distrito-sede tem uma area de $20 \mathrm{Km} 2 \mathrm{e}$ divide-se administrativamente em dois subdistritos. A área urbana dos três distritos foi definida pela Lei Municipal n. 817 .

Segundo estimativa da Agência Municipal do IBGE, no ano de 1969, o municipio de Botucatu possuia um total de 52.856 habitantes, sendo 7.600 na zona rural e 45.256 na zona urbana*.

Em 1968, a receita do município foi de Cr\$2.152.719,00 (excluída cotas-partes dos impostos federais e estaduais). Foram arrecadados para a União Cr\$2.136,00 e Cr\$12.612.877,00 para o Estado. A despesa municipal realizada foi de $\operatorname{Cr} \$ 3.232 .060,00$.

O município de Botucatu contava, em 1968, com 819 estabelecimentos comerciais varejistas, 21 atacadistas e 355 de prestaçōes de serviços. A agricultura, pecuária e silvicultura constituiram a principal atividade econômica, no município. No setor industrial contava com 105 indústrias, sendo 46 com mais de 5 funcionários, empregando aproximadamente 895 pessoas.

Botucatu contava com 9.998 domicílios em zona urbana e 1.132 em zona rural, em 1969. Em zona urbana, 6.919 residências estavam ligadas à rede de esgoto (sistema separador sem tratamento), enquanto 8.400 ligadas à rede de

* Comunicação pessoal. 
RAMOS, M. I. C. et al. - Análise de dados sócio-económicos e sanitáríos de famílias residentes no distrito-sede de Botucatu (São Paulo, Brasil), em 1969. Rev. Saúde públ., S. Paulo, 7: 161-79, 1973.

abastecimento de água. A captação principal é feita no Rio Pardo, sendo a água aduzida à estação de tratamento do tipo convencional. A cidade é também abastecida por poços profundos. A coleta de lixo era feita por 4 caminhões basculantes, sendo o resíduo depositado e queimado em terreno particular a $15 \mathrm{Km}$ da cidade. $O$ fornecimento de energia elétrica vem sendo feito pela Companhia Paulista de Força e Luz, sendo o número de ligações cerca de 8.965 , na cidade de Botucatu, em $1969 * 1$.

No ensino registravam-se 14 grupos escolares, 6 estabelecimentos de ensino secundário, um ginásio comercial (SENAC), um colégio comercial, 3 escolas normais, um estabelecimento de $1 .{ }^{\circ}$ ciclo industrial, e 2 estabelecimentos de ensino superior e um conservatório municipal $* 1$.

O setor saúde em Botucatu, compreendendo os sub-setores públicos $\mathrm{e}$ privados, caracterizava-se por um conjun. to de instituições públicas e privadas trabalhando sem seguir uma política explicitamente formulada e sem coordenação inter e mesmo intra-setorial. Na época havia cerca de 40 médicos, praticamente todos mantendo vínculo empregatício ou contratual com entidades de saúde. Poucos profissionais mantinham clínicas nos moldes tradicionais, com a maioria da clientela ligada a algum esquema de previdência social, sugerindo a pequena participação desse sub-setor privado não organizado no conjunto da atuação de saúde. $O$ sub-setor de saúde privado organizado compreendia os Hospitais de Misericórdia Botucatuense e Sorocabana, sem fins lucrativos, porém aumentando suas atividades com a venda de serviços (inclusive ao Instituto Nacional de Previdência Social - INPS). O sub-setor público federal estava representado pelo ambulatório médico do INPS. O sub-setor público estadual compreendia um conjunto de unidades de Saúde Pública ligadas a diferentes secretarias estaduais. A Secretaria da Saúde pertenciam o Centro de Saúde, o Dispensário de Tuberculose e o Dispensário de Dermatologia, os Postos de Puericultura ns. 1 e 2, o Laboratório Regional do Instituto Adolfo Lutz e a Delegacia Regional de Saúde. A Secretaria da Educação estavam ligados o Hospital das Clínicas da Faculdade de Ciências Médicas e Biológicas de Botucatu e o Serviço Dentário Escolar ${ }^{3}$.

$$
2 \text { - MATERIaI E MÉtodos }
$$

\section{1 - Area e amostragem}

A área de pesquisa compreendeu a zona urbana do distrito-sede de Botucatu. A unidade de amostragem foi a família, tendo sido selecionadas 305 famílias no processo de amostragem por duplo estágio. O sorteio foi casual para os quarteirões e sistemático para moradias.

\section{2 - Critérios para definição de status}

Para determinação de status sócio-econômico da população foram utilizados três critérios: instrução e ocupação do chefe de familia e renda familiar. A ocupação foi classificada em 5 grupos, segundo Hutchinson ${ }^{*}$.

No critério renda, foram considerados os rendimentos obtidos por todos os membros da família e classificados em 10 níveis desde 0 a mais de $\operatorname{Cr} \$ 2.100,00$.

* Agência Municipal do IBGE (Botucatu) - Comunicação pessoal. 
RAMOS, M. I. C. et al. - Análise de dados sócio-econômicos e sanitários de familias residentes no distrito-sede de Botucatu (Săo Paulo, Brasil), em 1969. Rev. Saúde puibl., S. Paulo, 7: 161-79, 1973.

Enquanto que, no critério instrução, foram considerados 5 níveis, inclusive a sua falta. A cada um dos níveis dos critérios foi dado um valor, variando de 1 a 10 pontos para renđa e ocupação e 1 a 5 para instrução. A soma dos valores nos três critérios (ocupação, renda e instrução) foi dividida e classificada em três níveis de status:

Status $A-25$ a 18 pontos

Status B -17 a 10 pontos

Status $\mathrm{C}-9$ a 2 pontos

\section{3 - Coleta de dados}

Foi feita através de formulários, contendo 30 questões abertas e fechadas. As familias foram entrevistadas por 85 alunos do $3 .^{\circ}$ ano de Medicina cabendo, a cada um, uma média de 3,5 famílias.

Antes do início do trabalho de campo foram tomadas as seguintes providências: a) informar a população e pedir colaboração quanto às entrevistas através de comunicados feitos por duas emissoras de rádios; b) atualizar a planta cadastral da zona urbana disponível, datada de 1959; c) pré-teste do formulário; d) orientação dos alunos quanto à técnica de abordagem e entrevistas; e) estabelecimento de critério para a fase de coleta de informações.

\section{4 - Métodos Estatísticos}

As provas de aderência foram feitas pelo $\mathrm{X}^{2}$ (Chakravarti et al 2 ). As comparações entre proporções multinominais foram realizadas pelo método de Goodman ${ }^{5}$. As comparações entre várias proporções correlacionadas foram testadas usando-se a estatística $Q$ de CoçHARAN 7. A associação entre duas variáveis foi estudada pelo $\mathrm{X}:$ (Chakravarti et al 2). A segunda interação em tabelas de $2 \times 2 \times 2$ foi testada pelo $\mathrm{X}^{2}$ com um grau de liberdade 2 . Comparações entre populações multinominais foram testadas pelo método de Goodnan 4. A homogeneidade de binomiais foi testada pela estatística T de Chakravarti et al. 2.

(*) significante ao nível de $5 \%$

(**) significante ao nível de $1 \%$

A ausência de asterisco indica não significância.

$$
3 \text { - RESUltados E COMENTARIOS }
$$

\section{1 - Estrutura Populacional}

As 305 famílias compreenderam 1349 indivíduos, sendo o número médio de habitantes por domicílio de 4,1. A sua distribuição segundo idade, sexo e estado civil encontra-se na Tabela 1, onde verificamos que a razão de masculinidade é de $98 / 100$ para a população total e 107/100 para a população solteira. Trata-se de uma população jovem e economicamente ativa com predominância de indivíduos abaixo de 49 anos (83\%).

Quanto ao status sócio-econômico, a população pesquisada distribuiu-se conforme Tabela 2.

Pouco mais da metade da população entrevistada situa-se no nível sócio-econômico mais baixo. Esta distribuição amostral sugere a distribuição populacional. 
RAMOS, M. I. C. et al. - Análise de dados sócio-econômicos e sanitários de famílias residentes no distrito-sede de Botucatu (São Paulo, Brasil), em 1969. Rev. Saúde públ., S. Paulo, 7: 161-79, 1973.

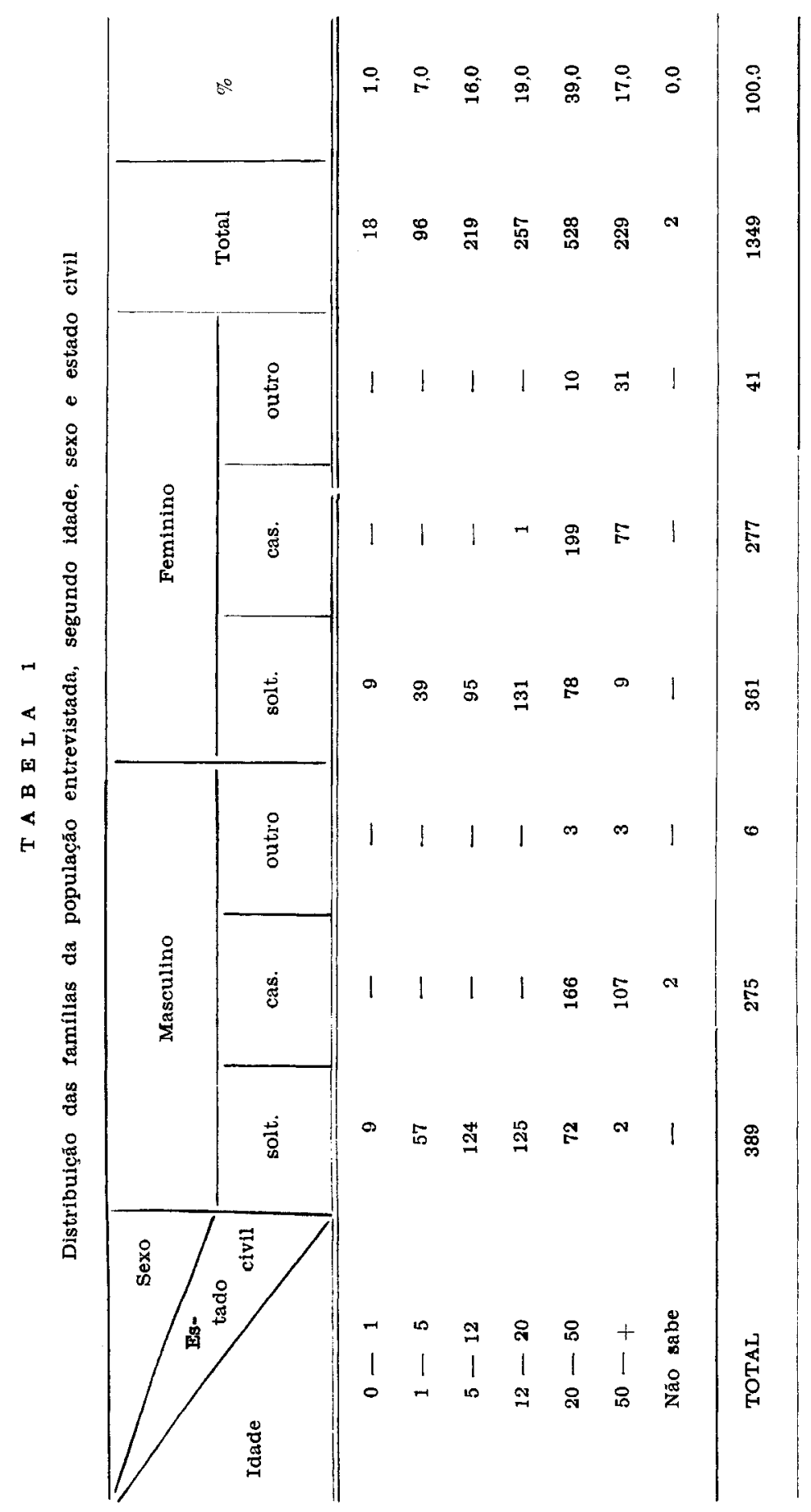


RAMOS, M. I. C. et al. - Análise de dados sóclo-econômícos e sanitários de famílias residentes no distrito-sede de Botucatu (São Paulo, Brasil), em 1969. Rev. Saúde pübl., S. Paulo, 7: 161-79, 1973.

\section{2 - Condições Sanitárias}

Abastecimento de água: - Em relação à procedência, apenas 2 pessoas não responderam constando para efeito de análise na Tabela 3,303 respostas. A água não encanada quase sempre provinha de poço. A água de beber e a água para outros usos eram da mesma procedência. Entre as pessoas, 77\% informaram a prática de tratamento domiciliar da água (filtro, fervura, moringa), enquanto $23 \%$ não a realizavam.

\begin{tabular}{c|cc} 
T A B E L A & \multicolumn{2}{c}{$\begin{array}{c}\text { Tistribuição } \\
\text { da } \begin{array}{c}\text { população } \\
\text { segundo status }\end{array}\end{array}$} \\
\hline Status & Número & $\%$ \\
\hline A & 31 & 10,0 \\
B & 107 & 35,0 \\
C & 167 & 55,0 \\
\hline
\end{tabular}

T A B E I A 3

Abastecimento de água

\begin{tabular}{l|c|c}
\multicolumn{1}{c|}{ Procedência } & \multicolumn{2}{|c}{ Freqüência } \\
\cline { 2 - 3 } & Absoluta & Relativa (\%) \\
\hline \hline $\begin{array}{c}\text { Encanada } \\
\text { (abastecimento público) } \\
\text { Năo encanada }\end{array}$ & 255 & 84,0 \\
& 48 & 16,00 \\
T O T A L & 303 & 100,0 \\
\hline
\end{tabular}

$$
\mathrm{x}^{2}=141,42^{*}
$$

A coleta do lixo pela Prefeitura foi referida por $80 \%$ dos entrevistados e negada por $20 \%$, num total de 305 respostas. A coleta era diária em $92 \%$ das 245 moradias e não diárias em 8\%.

Tendo sido variadas, duplas e de baixa frequiência, as respostas relativas ao destino do lixo foram agrupadas em duas classes, de acordo com condições consideradas adequadas ou não como demons tra a Tabela 4. Considerou-se como adequado o destino do lixo quando era incinerado ou enterrado. $O$ destino era inadequado quando o lixo era atirado em terreno baldio ou em depressões do solo, a céu aberto.

As réspostas relativas aos dois principais sistemas de destino dos dejetos distribuíram-se segundo mostra a Tabela 5 . Três respostas referiram eliminação direta na superfície do solo.

Os tipos de sistema com veiculação hídrica utilizados foram: $89 \%$ - esgoto, $7 \%$ de fossa, $2 \%$ de outros e $2 \%$ não souberam informar. 
RAMOS, M. I. C. et al. - Análise de dados sócio-econômicos e sanitários de famílas residentes no distrito-sede de Botucatu (São Paulo, Brasil), em 1969. Rev. Saúde públ., S. Paulo, 7: 161-79, 1973.

T A B E L A 4

Destino do Iixo, segundo coleta

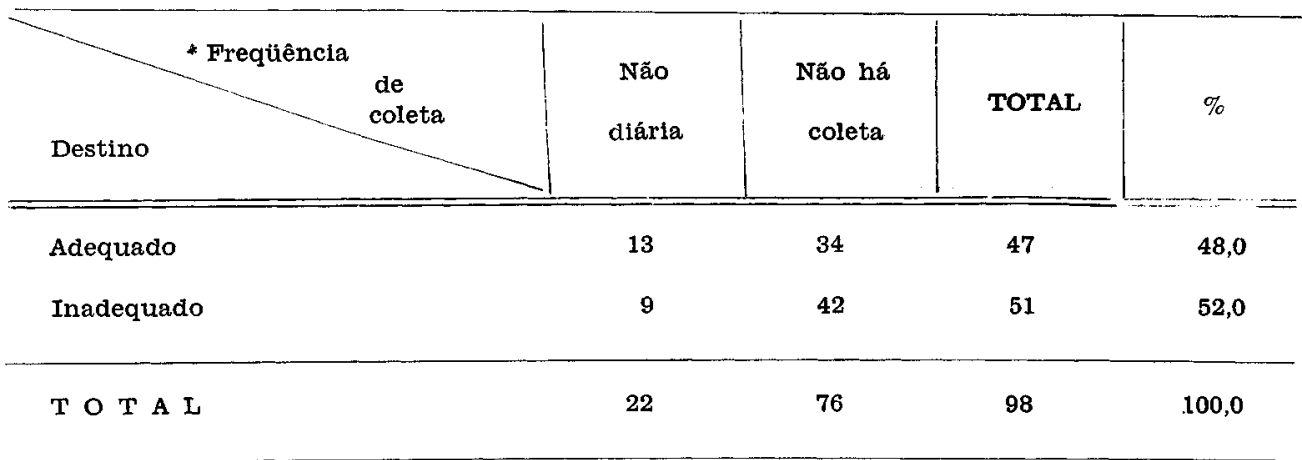

* excluída coleta diária

$\mathrm{x}^{2}=0,73$ (para os valores adequados -47 ; inadequados -51.$)$

T A B E L A 5

Destino dos dejetos: principais sistemas

\begin{tabular}{c|c|c}
\hline Destino & $\mathrm{n} .{ }^{\circ}$ & $\%$ \\
\hline Sem veiculaçāo hídrica & 50 & 17,0 \\
Com veiculação hídrica & 252 & 83,0 \\
\hline T O T A L & 302 & 100,00 \\
\hline
\end{tabular}

Com referência às condições de saneamento básico-abastecimento de água, coleta de lixo e destino dos dejetos em Botucatu verifica-se que:

- o abastecimento público de água, embora bom quanto à extensão (84\%) não atingiu toda a zona urbana; a prática de tratamento domiciliar da água, quase sempre por processos considerados adequados, é bastante difundida;
- a coleta diária do lixo atinge a maior parte dos domicílios urbanos; a metade das respostas da população entrevistada não servida pela coleta ou nos casos de coleta não diária, revelou destino inadequado, do ponto de vista sanitário;

- cerca de 70\% dos domicílios são servidos pela rede de esgotos; pequena parcela ainda utiliza a fossa seca ou sistemas inadequados com veiculação hídrica; não foi possível pesquisar se as fossas secas estavam dentro dos padrões sanitários.

\section{3 - Imunização}

Com as respostas obtidas em relação à aplicação, os tipos e doses de vacinas, apenas para crianças até 4 anos de idade inclusive, foram elaboradas as Tabelas $6,7,8,9$ e 10 (excluído um formulário). 
RAMOS, M. I. C. et al. - Análise de dados sócio-econômicos e sanitários de famillas residentes no distrito-sede de Botucatu (Sáo Paulo, Brasil), em 1969. Rev. Saúde públ., S. Paulo, 7: 161-79, 1973.

$\begin{array}{lllllll}\text { T A B E L A } & 6\end{array}$

Vacina Sabin - Aplicação e número de doses para crianças até 4 anos

\begin{tabular}{|c|c|c|c|c|c|c|}
\hline \multirow{2}{*}{$\begin{array}{l}\text { Total de crianças } \\
\text { até } 4 \text { anos }\end{array}$} & \multicolumn{3}{|c|}{ Doses } & \multirow{2}{*}{$\begin{array}{l}\text { Tomou mas } \\
\text { näo sabe o } \\
\text { n. }{ }^{\circ} \text { de doses }\end{array}$} & \multirow{2}{*}{$\begin{array}{l}\text { Năo } \\
\text { tomou }\end{array}$} & \multirow{2}{*}{$\begin{array}{l}\text { Não } \\
\text { respondeu }\end{array}$} \\
\hline & 1 & 2 & 3 & & & \\
\hline 113 & 4 & 12 & 29 & 56 & 5 & 7 \\
\hline
\end{tabular}

T A B E L A 7

Vacina $B C G$ - Aplicação e número de doses para crianças até 4 anos

\begin{tabular}{|c|c|c|c|c|c|c|}
\hline \multirow{2}{*}{$\begin{array}{l}\text { Total de crianças } \\
\text { até } 4 \text { anos }\end{array}$} & \multicolumn{3}{|c|}{ Doses } & \multirow{2}{*}{$\begin{array}{l}\text { Tomou mas } \\
\text { não sabe o } \\
\text { n. }{ }^{\circ} \text { de doses }\end{array}$} & \multirow{2}{*}{$\begin{array}{l}\text { Não } \\
\text { tomou }\end{array}$} & \multirow{2}{*}{$\begin{array}{l}\text { Não } \\
\text { respondeu }\end{array}$} \\
\hline & 1 & 2 & 3 & & & \\
\hline 113 & 8 & - & 5 & 61 & 24 & 15 \\
\hline
\end{tabular}

T A B E L A 8

Vacina Triplice - Aplicação e número de doses para crianças até 4 anos

\begin{tabular}{|c|c|c|c|c|c|c|}
\hline \multirow{2}{*}{$\begin{array}{l}\text { Total de crianças } \\
\text { até } 4 \text { anos }\end{array}$} & \multicolumn{3}{|c|}{ Doses } & \multirow{2}{*}{$\begin{array}{l}\text { Tomou mas } \\
\text { năo sabe o } \\
\text { n. de doses }\end{array}$} & \multirow{2}{*}{$\begin{array}{l}\text { Não } \\
\text { tomou }\end{array}$} & \multirow{2}{*}{$\begin{array}{l}\text { Não } \\
\text { respondeu }\end{array}$} \\
\hline & 1 & 2 & 3 & & & \\
\hline 113 & 12 & 2 & 15 & 40 & 31 & 13 \\
\hline
\end{tabular}

T A B E I A 9

Vacina Anti-variólica - Aplicação e número de doses para crianças até 4 anos

\begin{tabular}{c||c|c|c|c|c} 
Total de crianças & \multicolumn{2}{|c|}{ Doses } & $\begin{array}{l}\text { Tomou mas } \\
\text { não sabe o } \\
\text { no de doses }\end{array}$ & Não & tomou \\
\hline 113 & 1 & 2 & 2 Não & respondeu \\
\hline
\end{tabular}


RAMOS, M. I. C. et al. - Análise de dados sócio-econômicos e sanitários de famílias residentes no distrito-sede de Botucatu (São Paulo, Brasil), em 1969. Rev. Saúde pribl., S. Paulo, 7: 161-79, 1973.

T A B E L A 10

Distribuição das vacinas segundo tipo e aplicaçăo

\begin{tabular}{|c|c|c|c|c|}
\hline Aplicação Tipo & Varíola & Sabin & $\mathbf{B C G}$ & Triplice \\
\hline Vacinados & 43 & 79 & 63 & 50 \\
\hline Não vacinados & 41 & 4 & 20 & 33 \\
\hline TOTA L & 83 & 83 & 83 & 83 \\
\hline
\end{tabular}

$\mathbf{Q}=55,2 *$

$\mathrm{Na}$ tabela 10, são analisados em conjunto apenas as respostas de menores de 5 anos e sobre as quais se dispunham de informações sobre todas as vacinas.

Verifica-se pela análise das Tabelas 6 a 10 que a vacina mais referida foi a Sabin, fato previsto em virtude das campanhas bastante difundidas e da aceitação mais ampla quanto à proteção anti-poliomielite. Quanto à tríplice, é ainda elevado o número de crianças menores de 5 anos que não a receberam, fato que pode ser explicado pela insuficiência de trabalho educativo nesse sentido, tendo em vista que sua aceitação não é tão fácil como para a vacina Sabin. Outra hipótese estaria ligada à disponibilidade de fornecimento permanente dessa vacina pelos órgãos de saúde.

Deve ser comentado também o desconhecimento das mães quanto ao número de doses de vacinas aplicadas. Este achado pode relacionar-se diretamente à falta de informações sobre o valor das vacinas, e em última análise, à falta de educação sanitária eficiente.

Em relação à vacina anti-variólica, o número de crianças não vacinadas demonstrava a necessidade real que existia de se estender e sistematizar o programa de erradicação de variola no Estado, o que aliás ocorreu em Botucatu, três meses após o presente levantamento (fase de ataque de 7 a 13/12/1969).

As opiniões sobre vacinas foram extremamente variadas: assim, foram agrupadas e tabuladas segundo a Tabela 11.

T A B E LA 11

Opiniâo sobre as vacinas

Tipos de respostas N.O

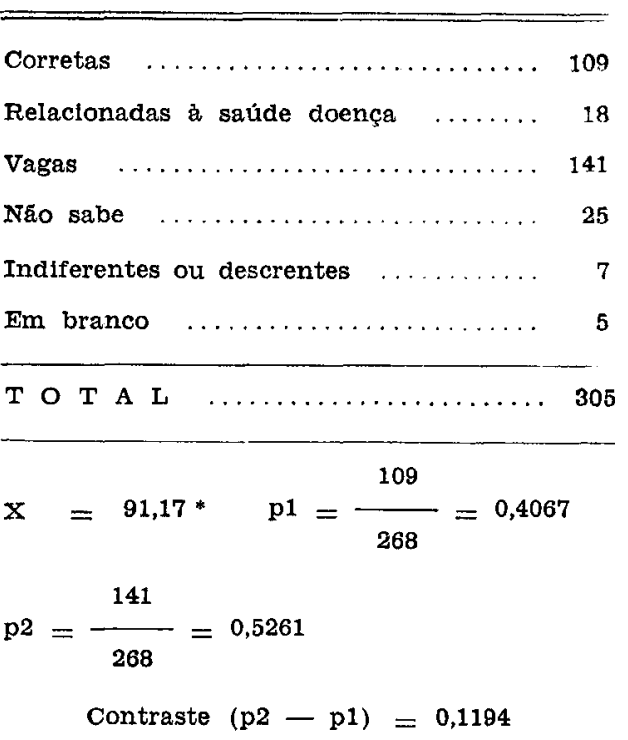


RAMOS, M. I. C. et al. - Análise de dados sócio-econômicos e sanitários de famílias residentes no distrito-sede de Botucatu (São Paulo, Brasil), em 1969. Rev. Saúde púbi., S. Paulo, 7: 161-79, 1973.

Foram consideradas como corretas aquelas que referiram claramente a ação preventiva frente às doenças infecciosas. As respostas que consideraram as vacinas "boas para a saúde" sem especificar sua ação protetora foram classificadas "relacionadas à saúde ou doença". Embora a diferença entre respostas corretas e vagas não seja significativa, evidenciou-se elevada proporção de pessoas que desconheciam o valor das vacinas provavelmente pelos motivos já discutidos anteriormente.
Quanto às fontes de informação sobre a necessidade de imunização, obtivemos respostas variadas e múltiplas que foram agrupadas na tabela 12. Em "outros meios" foram classificadas respostas que mencionavam, por exemplo, informações de vizinhos, colegas, familiares, farmacêuticos, cartório, etc. Instituição de saúde privada compreende respostas que mencionavam o médico particular como fonte de informação.

T A B E L A 12

Fontes de informação sobre vacinas

\begin{tabular}{lcc}
\hline Fonte de informação & Número & Total \\
\hline $\begin{array}{l}\text { Instituiçóes de saúde } \\
\text { Públicas } \\
\text { Privadas }\end{array}$ & 102 & 154 \\
\hline $\begin{array}{l}\text { Campanhas (divulgação) } \\
\text { Rádio } \\
\text { Escolas } \\
\text { Outras }\end{array}$ & 52 & \\
\hline $\begin{array}{l}\text { Outros meios } \\
\text { Năo sabe }\end{array}$ & 25 & 145 \\
\hline TOTAL & 49 & 49 \\
\hline
\end{tabular}

Verifica-se que as campanhas de divulgação somadas aos outros meios superam as instituições de saúde como fonte. No entanto, é preciso lembrar que as informações divulgadas pelo rádio, nas escolas, etc. provêm de instituições públicas que utilizam de todos os meios de comunicação disponiveis. $\mathrm{E}$ de se notar também que, em um número bem menor, os informes sobre imunizações foram dados pelo médico particular. Este dado levaria à suposição de que a classe médica ainda não estava convenientemente preocupada com medidas preventivas.

\section{4 - Demanda aos Serviços de Saúde 3.4.1 - Assistência ao parto}

Na Tabela 13 encontram-se distribuídas 
RAMOS, M. I. C. et al. - Análise de dados socio-econômicos e sanitários de famílias residentes no distrito-sede de Botucatu (São Paulo, Brasil), em 1969. Rev. Saúde públ., s. Paulo, 7: 161-79, 1973.

256 respostas relativas à questão sobre o local de nascimento e tipo de atendimento ao parto do filho mais novo, entre 305 entrevistadas (não responđeu: 1; não tiveram filhos: 46 ; respostas nulas: 2 ).

Analisando-se separadamente as respostas segundo o profissional e o local de atendimento verifica-se que as diferenças encontradas são significantes, o que indica, na ocasião, ser a parteira o profissional mais procurado para atenção ao parto. interessante assinalar também que a maior parcela dos partos feitos pela parteira foi domiciliar e que as curiosas realizavam mais partos do que as enfermeiras, tendo estas atuado quase que exclusivamente em ambiente hospitalar.

T A B E L A 13

Assistência ao parto do filho mais novo (Local de nascimento e tipo de profissional)

\begin{tabular}{|c|c|c|c|c|c|}
\hline $\begin{array}{l}\text { Local de } \\
\text { nascimento }\end{array}$ & Médtco & Enfermeira & Parteira & Curiosa & TOTAL \\
\hline Maternidade & 60 & 28 & 55 & - & 143 \\
\hline Domicilio & 5 & 3 & 76 & 29 & 113 \\
\hline $\mathbf{T} \mathbf{O} \mathbf{T} \mathrm{A} \mathbf{L}$ & 65 & 31 & 131 & 29 & 256 \\
\hline
\end{tabular}

Apesar da utilização dos serviços das curiosas ainda ocorrer, a proporção de partos por elas atendidos foi significantemente menor do que a proporção de partos feitos pelos outros profissionais, em nível domiciliar.

Quanto ao local de parto, em maternidade-143 e em casa-113 (total $=256$ ) não houve diferença significante $\left(X^{2}=3,28\right)$.

Foi solicitada às entrevistadas opinião a respeito do melhor local para assistên. cia ao parto. Na Tabela 14 estão expressos os resultados obtidos de respostas de 256 mulheres que tiveram filhos.

Comparando-se os dados relativos ao julgamento a respeito do que "acha melhor" com os dados da Tabela 13, nota-se
T A B E L A 14

Opinião sobre o melhor local para ter filhos

L o c a 1 N. 0

Maternidade 229

Em casa, não sabe ou não há diferença 27

T O T A L 256

uma nítida discrepância. Ao mesmo tempo em que 256 respostas, a grande maioria considera a maternidade como melhor local para o parto, no mesmo número de respostas, as diferenças entre as mulheres que informaram ter dado à luz em maternidade e em casa não foi 
RAMOS, M. I. C. et al. - Análise de dados sócio-econômicos e sanitários de famílias residentes no distrito-sede de Botucatu (São Paulo, Brasil), em 1969. Rev. Saúde públ., S. Paulo, 7: 161-79, 1973.

grande. Esta discrepância pode ser explicada pela idade das mães que responderam à questão.

Assim, cerca de $50 \%$ destas tinham idade na faixa de 34 a 53 anos, e portanto seus filhos mais novos nasceram possivelmente ainda numa época em que os recursos para assistência hospitalar ao parto eram menos disponíveis. Não foi possivel estabelecer a idade das mães na época do parto. Quanto às razões alegadas para justificar a preferência sobre o local do parto, a grande maioria referiu que a maternidade oferece condições de melhor assistência médica, segurança e conforto. As que preferiram o parto domiciliar apontaram razões de ordem econômica, costume e escassez de recursos assistenciais na época.

Entre 143 entrevistadas que tiveram parto hospitalar, ao serem indagadas também quanto ao tratamento feito no coto umbelical do recém-nascido, $\mathbf{5 9}$ não sabiam informar, não se lembravam ou não responderam. Oitenta e quatro res. ponderam ter feito curativos, dos quais apenas 3 eram inadequados. Foi considerado como curativo adequado aquêle em que se empregaram substâncias antissépticas e desinfetantes e curativo inadequado aquêle em que outras substâncias foram utilizadas. A grande maioria das mesmas pessoas deu uma opinião considerada adequada a respeito do que se deve passar no coto umbelical. Poucas declararam não saber e as respostas referindo curativo inadequado foram sete. Por outro lado, dentre 113 entrevistadas que deram à luz em casa, as respostas relativas ao que passaram no umbigo da criança foram distribuídas segundo Tabela 15 .

Vale ressaltar que foram mencionadas, por várias vezes entre as 17 respostas inadequadas, práticas bastante conheci-
T A B E L A 15

Tratamento empregado no coto umbelical

Não sabem ou năo se lembram .... 43

Uso de curativo adequado $\ldots \ldots \ldots \ldots \quad 49$

Uso de curativo inadequado $\ldots \ldots \ldots \ldots 17$

Nada passaram $\ldots \ldots \ldots \ldots \ldots \ldots \ldots$

T O T A L

$$
x_{2}^{2}=15,5 *
$$

das em medicina de "folk" principalmente em zonas rurais do Estado, como por exemplo, passar fumo, oleo de plantas de várias espécies, óleo de cozinha, teia de aranha embebida em óleo e mercúrio (prática mista) ou queimar com chama de vela ou ferro quente. As respostas referentes à opinião sobre como tratar o umbigo não divergiram muito em relação à prática utilizada. A utilização de métodos empíricos faz supor que as entrevistadas embora residindo em zona urbana seriam originárias de zona rural. Como informação complementar, deve ser assinalado que a ocorrência de tétano neo-natal, em Botucatu, segundo registro do Centro de Saúde, para os anos de 1967 e 1968 foi de 2 casos em cada ano.

Os dados seguintes, expressos na Tabela 16, referem-se às respostas obtidas quanto à assistência pré-natal, na última gravidez, na ausência de doença, distribuídas segundo o tipo de atenção ao parto (tipo de profissional). Verifica-se que as proporções das mulheres que não tendo feito pré-natal, foram atendidas por parteiras e sobretudo por curiosas, são mais elevadas (análise das diferenças entre as proporções pela estatística de Goodman ${ }^{5}$ ). 
RAMOS, M. I. C. et al. - Análise de dados sóclo-econômicos e sanitários de famílias resłdentes no distrito-sede de Botucatu (São Paulo, Brasil), em 1969. Rev. Saúde públ., S. Paulo, 7: 161-79, 1973.

\author{
T A B E L A 16
}

Assistência pré-natal na última gravidez, segundo o tipo de atenção ao parto

\begin{tabular}{|c|c|c|c|c|}
\hline \multirow{2}{*}{$\begin{array}{l}\text { Atenção ao } \\
\text { último parto }\end{array}$} & \multicolumn{2}{|c|}{$\mathbf{N} \quad \bar{A} \quad \mathbf{O}$} & \multirow{2}{*}{$\mathbf{S} \mathbf{I} \mathbf{M}$} & \multirow{2}{*}{$\mathbf{T} O \mathbf{T} A \mathbf{L}$} \\
\hline & N. 0 & $\%$ & & \\
\hline Médico & 12 & 18,0 & 53 & 65 \\
\hline Enfermeira & 8 & 26,0 & 23 & 31 \\
\hline Parteira & 58 & 44,0 & 73 & 131 \\
\hline Curiosa & 23 & 79,0 & 6 & 29 \\
\hline TOTAL & 101 & - & 155 & 256 \\
\hline
\end{tabular}

Para a elaboração da Tabela 17, que informa sobre o número de vezes em que 155 entrevistadas procuraram serviço pré-natal não estando doentes, foram excluídas 14 respostas vagas.

$$
\begin{array}{lllllll}
\text { T } & \text { A } & \text { B } & \text { E } & \text { L } & \text { A } & 17
\end{array}
$$

Demanda de serviços médicos na última gestação na ausência de doença: número de vezes.

\begin{tabular}{|c|c|c|c|}
\hline & & 1 & \\
\hline $\begin{array}{l}\text { Pelo menos } \\
3 \text { vezes }\end{array}$ & $\begin{array}{c}\text { De } 4 \text { a } 6 \\
\text { vezes }\end{array}$ & 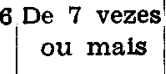 & T O T A L \\
\hline $\begin{array}{c}\mathbf{4 5} \\
\mathrm{p} 1=0,32\end{array}$ & $\begin{array}{c}30 \\
\mathrm{p} 2=0,21\end{array}$ & $\begin{array}{c}66 \\
\mathrm{p} 3=0,47\end{array}$ & 141 \\
\hline
\end{tabular}

$\mathrm{x}_{2}^{2}=14,67 * \quad$ Contrastes (diferenças entre proporçōes) :

$$
\begin{aligned}
& \mathrm{p} 1-\mathrm{p} 2=0,11 \\
& \mathrm{p} 1-\mathrm{p} 3=0,15 \\
& \mathrm{p} 2-\mathrm{p} 3=0,26 *
\end{aligned}
$$

Quanto ao número de vezes em que as entrevistadas procuraram serviços médicos durante a gravidez, observa-se não haver diferença significante na distribuição das que procuraram no menor e maior número de vezes, o que poderia indicar uma razoável tendência para inobservância da frequiência recomendada, entre aquelas que demandaram ao serviço pré-natal.

\subsection{2 - Sistemas Paralelos}

O conhecimento da população entrevistada sobre profissionais da medicina empírica é constatado na Tabela 18.

Fica evidenciado, também conforme a Tabela 19, a maior utilização dos serviços dos diferentes profissionais pela população de baixo nível sócio-econômico, recaindo a preferência sobre o benzedor.

\subsection{3 - Sistemas institucionais de prestação de serviços}

Dentre 305 familias apenas $86(28 \%)$ procuraram única e exclusivamente um recurso público ou privado, para indicação terapêutica, segundo Tabelas 20 e 21. As 219 famílias restantes utilizavam-se de recursos de diferentes serviços. 
RAMOS, M. I. C. et al. - Análise de dados sócio-econômicos e sanitários de famillas residentes no distrito-sede de Botucatu (São Paulo, Brasil), em 1969. Rev. Saúde públ., S. Paulo, 7: 161-79, 1973.

T A B E L A 18

Conhecimento sobre profissionais de medicina empírica, segundo status

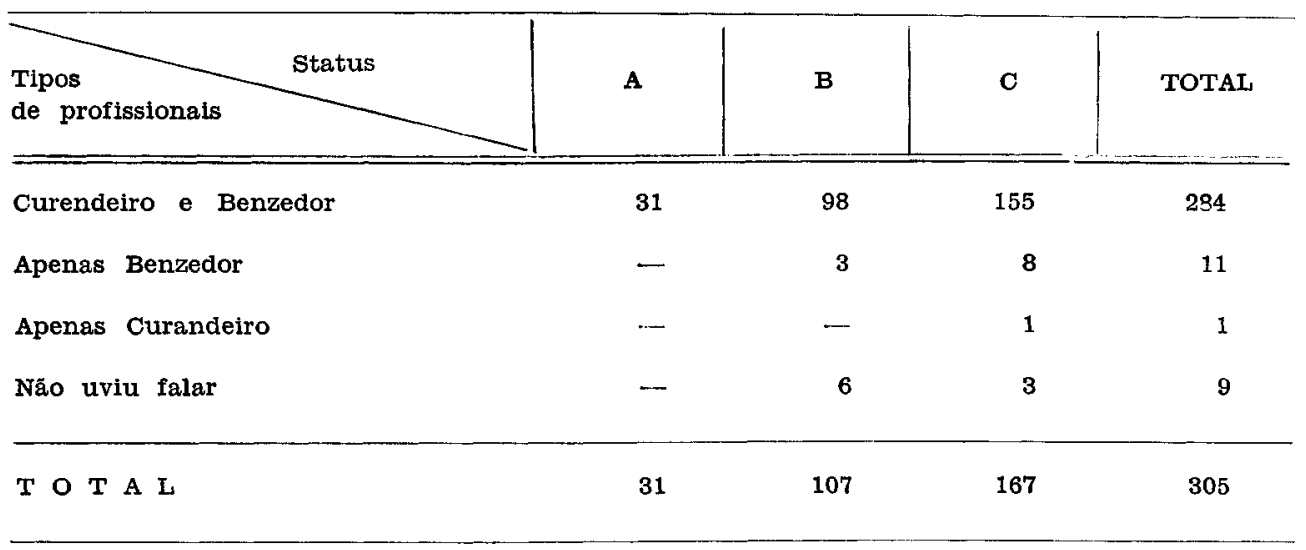

T A B E L A 19

Utilizaçăo de diferentes tipos de profissionais de medicina empírica, segundo status

\begin{tabular}{|c|c|c|c|c|}
\hline Status & Benzedor & Curandeiro & $\begin{array}{l}\text { Benzedor e } \\
\text { curandeiro }\end{array}$ & TOTAL \\
\hline A & 8 & 0 & 0 & 8 \\
\hline $\mathbf{B}$ & 26 & 2 & 5 & 33 \\
\hline $\mathbf{C}$ & 58 & 11 & 13 & 82 \\
\hline TOTAL & 92 & 13 & 18 & 123 \\
\hline
\end{tabular}

T A B E L A 20

Utilização de serviços públicos para indicação terapêutica segundo status

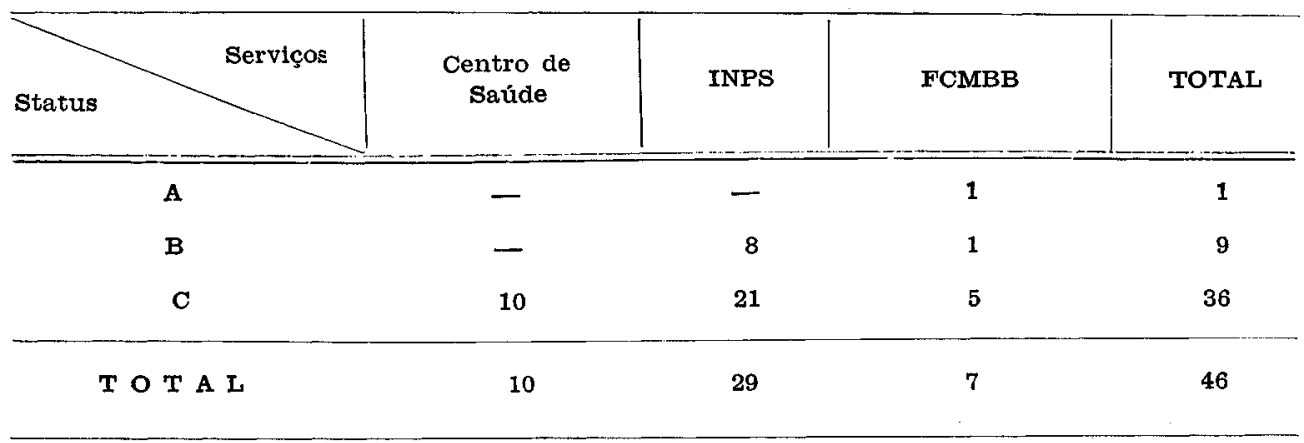


RAMOS, M. I. C. et al. - Análise de dados sócio-econômicos e sanitários de famílias residentes no distrito-sede de Botucatu (São Paulo, Brasil), em 1969. Rev. Saúde públ., S. Paulo, 7: 161-79, 1973.

T A B E L A 21

Utilização de serviços privados para indicação terapêutica segundo status

\begin{tabular}{|c|c|c|c|c|}
\hline Status & $\begin{array}{c}\text { Médico } \\
\text { Particular }\end{array}$ & $\begin{array}{c}\text { Santa } \\
\text { Casa }\end{array}$ & $\begin{array}{l}\text { Local de } \\
\text { Tabalho }\end{array}$ & TOTAL \\
\hline $\mathbf{A}$ & 7 & - & - & 7 \\
\hline B & 16 & - & - & 16 \\
\hline C & 14 & 2 & 1 & 17 \\
\hline $\mathbf{T} O \mathbf{T} \mathbf{A} \mathbf{L}$ & 37 & 2 & 1 & 40 \\
\hline
\end{tabular}

Os resultados globais expressos na Tabela 20 e 21 sugerem não haver diferença entre a utilização dos serviços públicos ou privados; quando analisados os 2 tipos de serviços, parece haver certa relação entre status e utilização de cada um dos serviços. É importante ressaltar que todas respostas, segundo status, correspondem à distribuição das proporções do status na população entrevistada. A maior parte (219 famílias) tende a procurar mais de um recurso, fato esperado em função da multiplicidade de serviços de assistência médica sem definição de clientela, no distrito-sede de Botucatu.

Nas respostas referentes à utilização de todos os tipos de serviços, Tabela 22, houve mais de uma opção no que se refere aos serviços públicos e foram considerados serviços paramédicos aqueles exercidos pelo farmacêutico.

Dentre as famílias que procuraram o serviço público, isolada ou associadamente, o predomínio de respostas do status C, além de corresponder à sua maior proporção na amostra populacional, pode ser devido ao maior contingente de população previdenciária nesse status.

Apesar da grande oferta de serviços médicos, a procura da farmácia (serviços paramédicos) para indicação terapêutica, era elevada, especificamente pelos indivíduos da classe $\mathrm{C}$, segundo mostra a Tabela 22. Este fato pode ser atribuido à maior facilidade de acesso aos serviços paramédicos do que aos serviços médicos, além de padrões culturais estabelecidos. Note-se que nenhum individuo de classe A referiu procura exclusiva dos serviços paramédicos.

Conforme Tabela 23, da população pesquisada, $75 \%$ eram beneficiários do INPS, Instituto de Assistência Médica do Servidor Público (IAMSP) e Instituto de Pensões e Aposentadoria do Servidor (IPASE), enquanto $23 \%$ não eram previdenciários. Cabe ressaltar que, em algumas famílias, seus membros pertenciam a diferentes institutos e que também no distrito-sede há predomínio de atividades do setor terciário.

Dessa população de 229 previdenciários, 168 famílias ou $73 \%$ recorriam ao INPS através dos Hospitais da Sorocabana e Misericórdia, enquanto 61 (27\%) não recorriam a serviços previdenciários, segundo a Tabela 23 . 
RAMOS, M. I. C. et al. - Análise de dados sócio-econômicos e sanitários de famílias residentes no distrito-sede de Botucatu (São Paulo, Brasil), em 1969. Rev. Saúde públ., S. Paulo, 7: 161-79, 1973.

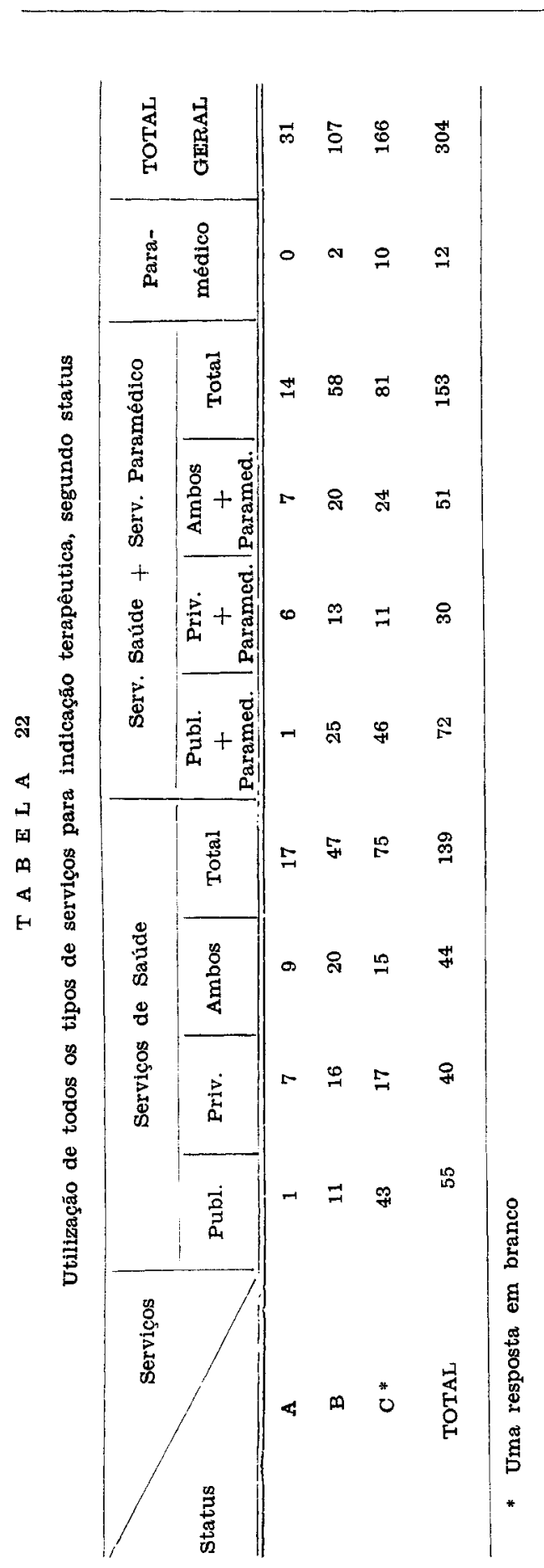

T A B E L A 23

População previdenctária e não previdenciária

\begin{tabular}{|c|c|c|}
\hline Filiados & N. ${ }^{0}$ & $\%$ \\
\hline INPS & 200 & 65,0 \\
\hline$\ldots \ldots \ldots$ & 21 & 8.0 \\
\hline INPS e IAMSP & 7 & 2,0 \\
\hline$\ldots \ldots \ldots$ & 1 & 0,0 \\
\hline SUB-TOTAL & 229 & 75.0 \\
\hline Não filliados & 70 & 23,0 \\
\hline Nulos & 6 & 2,0 \\
\hline GERAL & 305 & 1000 \\
\hline
\end{tabular}

T A B E L A 24

Demanda aos serviços previdenciários segundo vínculo previdenciário

\begin{tabular}{l|r|r|r} 
& Sim & Não & TOTAL \\
\hline Vínculo & & 39 & 200 \\
INPS & 161 & - & 7 \\
INPS/IAMSP & 7 & 21 & 21 \\
IAMSP & - & 1 & 1 \\
IPASE & - & 61 & 229 \\
\hline TOTAL GERAL & 168 & 61
\end{tabular}

Considerando-se aquelas famílias com vinculo ao INPS (207) constata-se que 39 não procuravam esse serviço $(19 \%)$ e dos 168 restantes, 29 procuravam exclusivamente os serviços do INPS (17\%), conforme assinalado na Tabela 20. Este fato, que revela baixa procura dos recursos previdenciários, pode ser explicado pela utilização de outros serviços, 
RAMOS, M. I. C. et al. - Análise de dados sócio-econômicos e sanitários de famílias residentes no distrito-sede de Botucatu (Săo Paulo, Brasil), em 1969. Rev. Saúde públ., S. Paulo, 7: 161-79, 1973.

reflexo da situação dispersiva dos recursos de assistência médica.

Dessas 207 famílias, 91 ou seja $44 \%$ conforme Tabela 25 consideraram que os serviços prestados devem sofrer modificações quanto à oferta e qualidade principalmente, $o$ que é expresso em termos de "atendimento rápido", "aumentar número de médicos", "melhor e mais atendimento".

T A B E L A 25

Necessidade de modificaçóes quantitativas e qualitativas do atendimento pelo INPS

\begin{tabular}{l|c|c}
\hline Respostas & N.o & $\%$ \\
\hline Sim & 91 & 44,0 \\
Não & 71 & 35,0 \\
Não sabe & 34 & 16,0 \\
Em branco & 10 & 5,0 \\
\hline T O T A L & & \\
\hline $\mathrm{X}_{2}^{2}=25,4^{\prime} 7^{*}$ & 207 & 100,0 \\
\hline
\end{tabular}

Embora pareçam ser pouco utilizados os serviços do Centro de Saúde, (conforme a Tabela 20) $63 \%$ da população o fez, conforme Tabela 26, com maior frequiência para fins preventivos, principalmente para imunização. Tendo em vista que a imunização é atividade desenvolvida regularmente pelo Centro de Saúde e não havendo na ocasião campanhas domiciliares era de se esperar, portanto, que a cobertura de vacinaçóes para a população tenha sido baixa, acrescentando-se ainda a ausência de programa de complementação de doses.

Trinta e sete por cento da população que dispensou os serviços do Centro de
Saúde procurou outros recursos ou informou "não ter necessidade", revelando sempre a percepção de que os serviços do Centro de Saúde destinam-se exclusivamente ao atendimento à criança não sido encontrada associação significante entre status e razões alegadas. $O$ motivo principal da não necessidade ou da pequena procura ao Centro de Saúde, foi o fato do mesmo se restringir somente as atividades de atenção à criança, na época.

T A B E L A 26

Demanda ao Centro de Saúde com finalidades preventivas, segundo o status.

\begin{tabular}{lr|c|c}
\hline Freqüência & Sim & Não & TOTAL \\
\hline A & 18 & 13 & 31 \\
B & 68 & 39 & 107 \\
C & 105 & 62 & 167 \\
\hline T O T A L & 191 & 114 & 305 \\
\hline X 2 & & \\
\hline $2=0,32$
\end{tabular}

T A B E L A 27

Tipos de dificuldades em relação aos cuidados de saúde

\begin{tabular}{|c|c|c|}
\hline Tipos de dificuldades & N. ${ }^{\circ}$ & $\%$ \\
\hline Financeiras $\quad \ldots$. & 35 & 37,0 \\
\hline $\begin{array}{c}\text { Estruturais e } \\
\text { nizacionais }\end{array}$ & 54 & 57,0 \\
\hline$\ldots \ldots \ldots$ & 6 & 6,0 \\
\hline $\mathrm{T}$ O $\mathrm{T}$ A L $\ldots$ & 95 & 100,0 \\
\hline
\end{tabular}

$\mathrm{x}_{2}^{2}=33,47^{*}$ 
RAMOS, M. I. C. et al. - Análise de dados sócio-econômicos e sanitários de famílias residentes no distrito-sede de Botucatu (São Paulo, Brasil), em 1969. Rev. Saúde públ., S. Paulo, 7: 161-79, 1973.

Embora 37\% tenham indicado dificuldades econômicas para cuidado de saúde, a maior parte $(57 \%)$ referiu-se a falhas decorrentes da estrutura e organização do sistema de saúde, expressas em termos de "falta de especialistas, de serviços de emergência, filas de espera e qualidade de atenção médica" (Tabela 27).

$$
4-\mathrm{C} O \mathrm{~N} \text { C L U S Õ E S }
$$

- Concentrando-se a população em baixo estrato sócio-econômico e sendo jovem, todo programa em saúde deve ser prioritariamente dirigido a essa população.

- Embora as condições de saneamento fossem razoáveis quantitativamente, dever-se-ia procurar atingir um nível mais elevado e o maior esforço deveria ser dispendido na elevação do padrão qualitativo.

- A necessidade de intensificar o trabalho educativo em saúde foi atestada pelo desconhecimento do esquema de vacinação e de seu caráter preventivo
- Os recursos existentes eram numericamente suficientes havendo porém inadequada utilização $\mathrm{e} / \mathrm{ou}$ procura simultânea de vários profissionais e instituições, donde ficou patente a necessidade de coordenação de órgãos assistenciais para saúde, evitando a duplicação dos recursos existentes.

- Com relação à utilização do Centro de Saúde, ficou constatado que era pequena a demanda a este serviço sendo feita quase sempre para imunização principalmente nas faixas etárias mais baixas. Desta forma, verificou-se a necessidade de modificar a percepção quanto ao Centro de Saúde, através do desenvolvimento adequado das demais atividades médico-sanitárias programadas e de trabalho educativo sistemático, junto à população.

\section{AGRADECIMENTOS}

Aos alunos do $3 .^{\circ}$ ano de Medicina da Faculdade de Ciências Médicas e Biológicas de Botucatu (1969) pela coleta de dados.

Ramos, M. I. C. et al. - [Socioeconomic and sanitary survey in families living in the Botucatu County (S. Paulo, Brazil) in 1969] Rev. Saúde públ., S. Paulo, 7: 161-79, 1973.

SUMmaRy: In 1969 , a survey was carried out concerning the socioeconomical and sanitary aspects of the inhabitants in Botucatu County (S. Paulo, Brazil). Three hundred and five families were interviewed. Data involving the composition of the family, age of the membres, occupation, salary, sanitary conditions of their houses, knowledge about vaccination and their opinions about health services were collected and analysed. Some results were associated with the socioeconomic status of the families. The basic sanitation conditions were adequate and of reasonable standard. Knowledge about the value of vaccination as well as the required doses were vague Resources available for health were fragmented on indication of the lack of coordenation among the health services. Health centers were mainly sought for the care of children, on indication of the 
RAMOS, M. I. C. et al. - Análise de dados sócio-econômicos e sanitários de famílias residentes no distrito-sede de Botucatu (São Paulo, Brasil), em 1969. Rev. Saúde públ., S. Paulo, 7: 161-79, 1973.

population's low degree of knowledge about their purposes. It was concluded that an improvement of the community viewpoints about medical-sanitary aspects is desired and systematic health educaiton programs are necessary and urgent.

Uniterms: Socioeconomic survey (Botucatu, S. Paulo, Brazil)*; Sanitation * Health resortes *

\section{REFERENCIAS BIBLIOGRAFICAS}

1. BOLETIM ESTATISTICO. PREFETTURA MUNICIPAL DE BOTUCATU. Botucatu, 1970.

2. CHAKRAVARTI, I. M. et al. - Handbook of methods of applied statistics. New York, Wiley, 1967, v. 1, p-307.

3. FUNDAÇÃO DE RECURSOS HUMANOS PARA A SAÚDE. Departamento de Metodologia do Planejamento Diagnóstico setorial de saúde de Botucatu. Rio de Janeiro, Fundação Ensino Especializado de Saúde Pública, 1969. (Documento, 97)
4. GOODMAN, L. A. - Simultaneous confidence intervals for contrasts among multinomial populations. Ann. Math. Stat., 35:716-25, 1964.

5. GOODMAN, L. A. - On simultaneous confidence intervals for multinomial proportions. Technometrics, 7:247 -54, 1965.

6. HUTCHINSON, B. - Mobilidade e trabatho. Rio de Janeiro, Instituto Nacional de Estudos Pedagógicos, 1960.

7. SIEGEL, S. - Non parametric statistics. Tokyo, McGraw-Hill, 1956. 